\title{
Entendendo os Mecanismos Determinantes da Lesão Pulmonar Induzida pela Ventilação Mecânica*
}

\section{Understanding the Mechanisms of Ventilator-induced Lung Injury}

\author{
Liliane M. Nardelli', Cristiane S. N. B. Garcia², Caroline P Pássaro ${ }^{3}$, Patricia R. M. Rocco ${ }^{4}$
}

\section{RESUMO}

JUSTIFICATIVA E OBJETIVOS: A ventilação mecânica é considerada elemento básico de suporte de vida nas unidades de terapia intensiva e, indubitavelmente, essencial para os pacientes com lesão pulmonar aguda (LPA) e síndrome do desconforto respiratório agudo (SDRA). Estudos experimentais demonstraram que a ventilação mecânica (VM) com altos volumes e/ ou altas pressões pode exacerbar ou iniciar uma lesão pulmonar, denominada lesão pulmonar associada à VM (LPAV) ou lesão pulmonar induzida pelo ventilador

1. Aluna de Mestrado da Pós-Graduação em Fisiologia, Laboratório de Investigação Pulmonar, Instituto de Biofísica Carlos Chagas Filho da UFRJ

2. Doutora em Ciências pela UFRJ, Pós-Doutoranda do Laboratório de Investigação Pulmonar, Instituto de Biofísica Carlos Chagas Filho da UFRJ

3. Doutora em Ciências pela UFRJ, Laboratório de Investigação Pulmonar, Instituto de Biofísica Carlos Chagas Filho da UFRJ

4. Professora Associada da UFRJ; Doutora em Ciências pela UFRJ, Chefe do Laboratório de Investigação Pulmonar, Instituto de Biofísica Carlos Chagas Filho da UFRJ

*Recebido do Laboratório de Investigação Pulmonar, Instituto de Biofísica Carlos Chagas Filho, Universidade Federal do Rio de Janeiro (UFRJ), Rio de Janeiro, RJ

- Apoio Financeiro: Fundação Carlos Chagas Filho de Amparo à Pesquisa do Estado do Rio de Janeiro (FAPERJ), Conselho Nacional de Desenvolvimento Científico (CNPq), Programa de Apoio a Núcleos de Excelência (PRONEX-FAPERJ).

Apresentado em 30 de julho de 2007

Aceito para publicação em 25 de setembro de 2007

Endereço para correspondência:

Profa. Dra. Patricia Rieken Macedo Rocco

Laboratório de Investigação Pulmonar

Instituto de Biofísica Carlos Chagas Filho - C.C.S. - UFRJ

Edifício do Centro de Ciências da Saúde, Bloco G-014

Av. Carlos Chagas Filho, s/n

Cidade Universitária, Ilha do Fundão

21941-902 Rio de Janeiro, RJ

Fone: (21) 2562-6530 - Fax: (21) 2280-8193

E-mail: prmrocco@biof.ufrj.br

(C)Associação de Medicina Intensiva Brasileira, 2007
(LPIV), respectivamente, com aspecto histológico similar ao da LPA/SDRA.

CONTEÚDO: Realizou-se uma pesquisa sistemática dos artigos incluídos na MedLine e SciElo dos últimos 20 anos, que abordavam uma visão crítica dos principais mecanismos determinantes da LPIV. Dentre os principais mecanismos da LPAV/LPIV pode-se citar: volutrauma causado por hiperdistensão e expansão desigual das unidades alveolares em função de altas pressões transpulmonares ou volumes; aletectrauma resultante da abertura e fechamento cíclicos das vias aéreas distais e o biotrauma determinado pelo processo inflamatório conseqüente às estratégias ventilatórias lesivas adotadas.

CONCLUSÕES: Os mecanismos responsáveis pelo volutrauma, atelectrauma e biotrauma devem ser bem entendidos para que se possa evitar a lesão associada à ventilação mecânica.

Unitermos: Estresse Alveolar, Lesão Pulmonar Aguda, SDRA, Ventilação Mecânica

\section{SUMMARY}

BACKGROUND AND OBJECTIVES: Mechanical ventilation is considered a basic element of life support in the intensive care unit and is essential for patients with acute lung injury (ALI) and acute respiratory distress syndrome (ARDS). Experimental studies have demonstrated that mechanical ventilation with high volumes and/or high pressures can exacerbate (VALI) or induce lung injury (VILI) with histological aspect similar to ALI/ ARDS.

CONTENTS: This systematic review included the literature on MedLine and SciElo database published in the last 20 years. In this review, we will highlight the most recent data on the mechanisms of VILI. The main mechanisms of VILI are: volutrauma caused by overinflation and uneven expansion of the lungs due to high ventilation pressures or volumes; aletectrauma induced by shear forces generated during cyclic closure and re- 
opening of terminal airways; and biotrauma where the injury resulted from the release inflammatory mediators due to physical stresses associated with mechanical ventilation.

CONCLUSIONS: It is fundamental to understand the mechanisms related to volutrauma, atelectrauma, and biotrauma to avoid ventilator-associated lung injury.

Key Words: Acute Lung Injury, Alveolar Stress, ARDS, Mechanical Ventilation

\section{INTRODUÇÃO}

A ventilação mecânica (VM), ao longo dos últimos 40-50 anos, tornou-se indispensável modalidade terapêutica na síndrome do desconforto respiratório agudo (SDRA), promovendo oxigenação adequada e repouso da musculatura respiratória 1 . Contudo, nas últimas três décadas, tornou-se evidente que a VM pode exacerbar ou iniciar uma lesão pulmonar, denominada lesão pulmonar associada à VM (LPAV) ou lesão pulmonar induzida pelo ventilador (LPIV), respectivamente ${ }^{2}$.

A LPAV é um importante componente na patogênese da lesão pulmonar aguda (LPA)/SDRA ${ }^{3}$, podendo contribuir para a elevada taxa de mortalidade. Nesse contexto, constatou-se redução da mortalidade de pacientes com LPA/SDRA (31\%) ventilados com volumecorrente $\left(\mathrm{V}_{\mathrm{T}}\right)$ de $6 \mathrm{~mL} / \mathrm{kg}$ quando comparado com $\mathrm{V}_{\mathrm{T}}$ de $12 \mathrm{~mL} / \mathrm{kg}(40 \%)^{4}$.

A LPAV/LPIV resulta da interação complexa de diferentes forças mecânicas que atuam sobre o pulmão e depende da natureza e intensidade do estresse mecânico induzido pela $\mathrm{VM}^{5}$.

O objetivo do presente estudo foi descrever os diferentes mecanismos da LPIV/LPAV, bem como os seus fatores determinantes, contribuindo, desta forma, para o desenvolvimento de novas estratégias ventilatórias na LPA/SDRA.

\section{ALTERAÇÕES MORFOLÓGICAS}

As alterações histológicas observadas na LPAV/LPIV são similares àquelas observadas na SDRA ${ }^{6-8}$, e apresentam clara relação direta com a duração da VM e a intensidade do estímulo de lesão9. A VM com altas pressões transpulmonares acarreta lesão alveolar difusa caracterizada pela presença de membrana hialina, hemorragia alveolar e infiltração neutrofílica ${ }^{10}$. A microscopia eletrônica permite a visualização de lesões endoteliais e epiteliais em poucos minutos de VM com altas pressões nas vias aéreas ${ }^{6,7}$, caracteri- zadas por descontinuidade em pneumócitos tipo I11, desacoplamento das células endoteliais da membrana basal, rupturas endoteliais e edema alveolar ${ }^{5}$. Com a progressão da lesão, constata-se achados histológicos pulmonares, compatíveis com o estágio tardio da SDRA: proliferação de fibroblastos e de células epiteliais alveolares tipo $\mathrm{II}^{12}$.

\section{Edema Pulmonar}

Os pulmões apresentam algumas características intrínsecas que os protegem contra o acúmulo de líquido no espaço extravascular e o desenvolvimento de edema. Essa proteção é determinada por três componentes: (1) pressão de filtração capilar; (2) capacidade do espaço intersticial em reabsorver o líquido extravascular e (3) capacidade do sistema linfático pulmonar em transportar fluidos para fora do pulmão.

Uma vez que as pressões aplicadas ao espaço aéreo também repercutem nos capilares pulmonares, as pressões e os fluxos intravasculares devem ser considerados na evolução e/ou no desenvolvimento da LPIV. Mesmo em pulmões normais, a insuflação alveolar impõe estresse vascular aos capilares pulmonares ${ }^{10,13}$. Webb e Tierney demonstraram que a VM, por si só, pode induzir a formação de edema pulmonar, que se desenvolve mais rapidamente nos animais ventilados com pressão de pico inspiratória (PIP) de $45 \mathrm{cmH}_{2} \mathrm{O}$ do que com $30 \mathrm{cmH}_{2} \mathrm{O}^{10}$. Entretanto, a PIP menor ou igual a $30 \mathrm{cmH}_{2} \mathrm{O}$ também pode acarretar edema pulmonar ${ }^{14}$, sendo que uma lesão pulmonar preexistente pode atuar de forma sinérgica ${ }^{15}$.

Inicialmente, acreditava-se que o edema pulmonar induzido pela VM seria determinado por aumento na pressão transmural vascular ${ }^{10}$, que poderia elevar a pressão de filtração nos capilares extra-alveolares ${ }^{16} \mathrm{e}$ alveolares ${ }^{17}$. Entretanto, a análise microscópica do fluido do edema pulmonar evidenciou infiltrado eosinofílico e grande quantidade de proteínas, sugerindo uma alteração na permeabilidade da barreira alvéolo-capilar. Nesse contexto, estudos demonstraram aumento da permeabilidade a pequenos e grandes solutos ${ }^{18,19} \mathrm{e}$ anormalidades epiteliais e endoteliais evidenciadas na microscopia eletrônica ${ }^{6,7,11}$. Ademais, diferenças regionais na perfusão pulmonar e atelectasias podem acarretar aumento nas forças de filtração em determinadas unidades alvéolos-capilares, propiciando a formação de edema ${ }^{16}$.

A LPIV também compromete a habilidade do pulmão em reabsorver o edema. Inibição do transporte ativo de $\mathrm{Na}^{+}$e redução da atividade $\mathrm{Na}$, K-ATPase em pneumó- 
citos tipo II foram demonstradas em ratos submetidos à VM com hiperinsuflação ${ }^{20}$.

\section{Alteração do Sistema de Surfactante Pulmonar Endógeno}

$\mathrm{Na}$ fisiopatologia da LPIV, o surfactante ganha destaque sob duas formas: (1) a deficiência do surfactante exacerba as lesões induzidas pelo ventilador e (2) a própria VM pode afetar o sistema de surfactante, favorecendo o desenvolvimento da lesão pulmonar ${ }^{21}$.

O surfactante existe nos espaços aéreos alveolares sob duas formas estruturais principais: os grandes agregados, funcionalmente superiores e os pequenos agregados, funcionalmente inferiores. Mudanças nas proporções relativas destes agregados dentro dos espaços aéreos foram observadas em modelos animais de $\mathrm{LPA}^{22}$, em pacientes com SDRA ${ }^{23}$ e durante a VM com altos volumes ${ }^{21}$. As alterações no sistema de surfactante podem ser ainda maiores no pulmão com lesão preexistente submetido à VM com $\mathrm{V}_{\mathrm{T}}$ elevado ${ }^{24}$. A conversão dos agregados de surfactante induzida pelo $\mathrm{V}_{\mathrm{T}}$ elevado está relacionada a dois mecanismos: (1) alterações na área de superfície e (2) atividade aumentada de proteases nos espaços aéreos ${ }^{15}$.

A deficiência de surfactante aumenta a tensão superficial e pode contribuir para a gênese da LPIV de diversas formas: (1) os alvéolos e as vias aéreas ficam mais propensos ao colapso, (2) a expansão desigual das unidades pulmonares aumenta o estresse regional através do mecanismo de interdependência e (3) o aumento da pressão de filtração vascular promove a formação de edema.

\section{PRINCIPAIS DETERMINANTES DA LESÃO PULMO- NAR INDUZIDA PELO VENTILADOR}

\section{Barotrauma versus Volutrauma}

Por mais de 50 anos, as pesquisas mostraram que a VM com altas pressões nas vias aéreas acarretava lesões pulmonares, caracterizadas apenas por ruptura dos espaços aéreos e fuga de ar, produzindo muitas manifestações clínicas, que foram reconhecidas como barotrauma (pneumomediastino, enfisema subcutâneo, pneumotórax, pneumopericárdio, pneumoperitônio, enfisema subcutâneo intersticial) ${ }^{1}$. Portanto, a LPIV foi durante muito tempo sinônimo de barotrauma. Embora as conseqüências desses eventos macroscópicos tenham sido rapidamente reconhecidas, alterações morfológicas e fisiológicas mais sutis necessitaram mais tempo para serem descritas. Somente em 1974, Webb e Tierney demonstraram que a VM poderia ser responsável por lesão ultra-estrutural, independentemente da ocorrência de fuga de $\operatorname{ar}^{10}$. Este foi o primeiro estudo em animais sadios. Estudos subseqüentes confirmaram a lesão alveolar difusa induzida pela PIP alta ${ }^{6,25}$.

Entretanto, vale ressaltar que a pressão absoluta nas vias aéreas, por si só, não é lesiva. Isso foi confirmado pela observação de que tocadores de trompete comumente atingem pressões de vias aéreas de $150 \mathrm{cmH}_{2} \mathrm{O}$ sem desenvolverem lesão pulmonar ${ }^{26}$. Adicionalmente, evidências experimentais indicaram que o grau de insuflação pulmonar parece ser mais importante na gênese da lesão pulmonar do que o nível de pressão. $A$ contribuição relativa da PIP e do $V_{T}$, na lesão pulmonar, foi avaliada primeiramente em ratos sadios ventilados com limitação do movimento tóraco-abdominal ${ }^{7}$. Uma PIP alta $\left(45 \mathrm{cmH}_{2} \mathrm{O}\right)$ sem $\mathrm{V}_{\mathrm{T}}$ elevado não produziu lesão pulmonar. Entretanto, animais ventilados sem restrição do movimento tóraco-abdominal (alcançando $\mathrm{V}_{\mathrm{T}}$ alto), por pressão positiva ou negativa, desenvolveram lesão pulmonar grave. Esses achados foram confirmados em outras espécies animais ${ }^{27,28}$, o que gerou a definição do termo volutrauma.

Os pacientes com SDRA são mais susceptíveis à hiperdistensão alveolar, principalmente, quando submetidos à $\mathrm{VM}$ convencional com $\mathrm{V}_{\mathrm{T}}$ alto (10 a $\left.15 \mathrm{~mL} / \mathrm{kg}\right)$; já que o número de unidades pulmonares disponíveis para serem ventiladas é reduzido em função de acúmulo de líquido, consolidação e atelectasia ${ }^{29}$. A ventilação com baixo $V_{T}$ reduziu a taxa de mortalidade nos pacientes com SDRA ${ }^{4}$, porém isso não significa afirmar que um $V_{T}$ de $6 \mathrm{~mL} / \mathrm{kg}$ é seguro; mas que ele implica em melhor prognóstico do que $\mathrm{V}_{\mathrm{T}}$ de $12 \mathrm{~mL} / \mathrm{kg}$.

\section{Atelectrauma}

A lesão pulmonar também pode ser causada pela VM em baixos volumes pulmonares ao final da expiração. Esta lesão acredita-se estar relacionada à abertura e ao fechamento cíclicos de vias aéreas distais, ductos e unidades alveolares (daí o termo atelectrauma). O recrutamento e/ou desrecrutamento repetido das unidades pulmonares distais pode gerar um estresse de cisalhamento local aumentado, principalmente se o evento se repetir a cada ciclo respiratório. Durante a abertura das vias aéreas, observa-se a presença de dois tipos de estresse: um com ação perpendicular à superfície (estresse tensile) e outro paralelo à superfície de ação (estresse de cisalhamento) ${ }^{5}$. 


\section{Biotrauma}

O biotrauma descreve um processo de lesão onde forças biofísicas podem alterar a fisiologia normal das células pulmonares, induzindo aumento dos níveis de mediadores inflamatórios ${ }^{30}$ e promovendo o reparo/remodelamento do tecido. Estudos clínicos e experimentais demonstraram que as estratégias ventilatórias lesivas podem iniciar ou perpetuar uma resposta inflamatória local e sistêmica, que, por sua vez, pode contribuir significativamente para a $\mathrm{DMOS}^{30-32}$. Várias evidências apontam para isso, incluindo: (1) evidência histológica de infiltração neutrofílica; (2) níveis elevados de citocinas no fluido do lavado broncoalveolar e na circulação sistêmica ${ }^{31}$. Tremblay e col. descreveram que, após $2 \mathrm{~h}$ de $\mathrm{VM}$ com $\mathrm{V}_{\mathrm{T}}$ elevado, os níveis de TNF (fator de necrose tumoral)- $\alpha$ e IL (interleucina)- 6 aumentaram ${ }^{32}$. Entretanto, Ricard e col., não detectaram incremento dos níveis de TNF- $\alpha$ e da MIP (proteína inflamatória de macrófago)-2 no fluido do lavado broncoalveolar de pulmões sadios ventilados com $\mathrm{V}_{\mathrm{T}}$ alto $(42 \mathrm{~mL} / \mathrm{kg})$, por $2 \mathrm{~h}$, mas notaram um aumento significativo no nível de IL1- $\beta^{33}$. Adicionalmente, a VM com $V_{T}$ elevado e PEEP igual a zero acarretou lesão pulmonar e liberação de medidores inflamatórios ${ }^{34}$, ao contrário do observado durante a estratégia ventilatória protetora com baixo $V_{T}$ e PEEP alta ${ }^{35}$. Nesse contexto, vários estudos mostraram que a ventilação protetora promoveram redução dos níveis de IL-1, IL-6, IL-10 e TNF- $\alpha$, reforçando o fato de que a VM tem influência significativa na liberação de mediadores pró e antiinflamatórios no pulmão, podendo desempenhar um papel na gênese e perpetuação da resposta inflamatória local e sistêmica.

\section{OUTROS POSSÍVEIS DETERMINANTES DA LESÃO PULMONAR INDUZIDA PELO VENTILADOR}

\section{Freqüência Respiratória}

A LPIV também pode ser induzida pelo estresse mecânico cíclico determinado por elevadas freqüências ${ }^{36,37}$. Altas freqüências ventilatórias podem acarretar microfraturas no parênquima pulmonar, que aumentam em número e se propagam a cada ciclo até que o traço de fratura seja grande o suficiente para proporcionar falência tecidual ${ }^{36}$. Em volumes pulmonares normais, a membrana alvéolo-capilar suporta certa carga de estresse devido à capacidade das células epiteliais e endoteliais se adaptarem a um determinado grau de deformação, sem sofrer lesão. Entretanto, em vigência de lesão pulmonar subjacente e estratégias ventilatórias lesivas, a redução da FR pode oferecer uma proteção adicional ao desenvolvimento da LPIV ${ }^{36,37}$.

\section{Relação Tempo Inspiratório e Tempo Expiratório (I:E) Invertida}

A VM com relação I:E invertida (IRV) era usada em pacientes com SDRA para proporcionar redução da PEEP e/ou da PIP e melhorar a oxigenação arterial ${ }^{38}$. Adicionalmente, acreditava-se que a IRV poderia proporcionar uma distribuição de ar mais homogênea com menor risco de hiperdistensão alveolar. Entretanto, estudos experimentais ${ }^{39}$ demonstraram que o tempo inspiratório aumentado pode exacerbar a lesão pulmonar, piorando a relação ventilação-perfusão, reduzindo a complacência pulmonar e aumentando o edema pulmonar ${ }^{39}$.

\section{Fluxo Aéreo Inspiratório}

O fluxo aéreo inspiratório é também um importante determinante de estresse no pulmão. O fluxo inspiratório elevado aumenta o estresse tensile, resultando em transmissão de energia cinética para as estruturas subjacentes, e o estresse de cisalhamento, distorcendo o parênquima e deformando a superfície epitelial ${ }^{40}$. Durante a VM com PIP alta, foi demonstrado que o fluxo aéreo elevado causa lesão microvascular pulmonar ${ }^{41}$. Subseqüentemente, foi verificado efeito protetor da redução do fluxo inspiratório na LPA durante a ventilação com PIP elevada, resultando em menor grau de shunt, lesão histológica, infiltrado pulmonar neutrofílico e edema alveolar ${ }^{42}$.

\section{Resposta Celular às Forças Mecânicas}

Os pulmões, durante a VM, são submetidos a forças mecânicas anormais, que podem acarretar efeitos deletérios aos seus componentes celulares, alterando estrutura, função e metabolismo ${ }^{43}$.

Fisiologicamente, a pressão transpulmonar é transmitida igualmente para todas as regiões pulmonares. Contudo, no pulmão heterogêneo de pacientes com LPA/ SDRA, a pressão transpulmonar não é distribuída uniformemente, gerando estresses regionais excessivos ${ }^{5}$. A mecanotransdução é a conversão de um estímulo mecânico, tal como a deformação celular, em alterações bioquímicas e biomoleculares intracelulares. Ainda não está bem compreendido como as forças mecânicas são captadas pelas células (mecanosensores) e convertidas em sinais bioquímicos e biológicos ${ }^{5,44} . \mathrm{Na}$ LPIV, três mecanismos primários parecem participar da mecanotransdução: (1) canais estiramentos-sensíveis; 
(2) alterações da integridade da membrana plasmática, e/ou (3) alterações na conformação direta das moléculas associadas à membrana ${ }^{5}$.

A sinalização intracelular inclui a geração de segundo mensageiro, a ativação de quinases protéicas específicas, a fosforilação e a ativação de moléculas de sinalização, a amplificação dos sinais por cascatas enzimáticas e a modulação de expressão gênica ${ }^{5,44}$. No núcleo, as forças biofísicas podem exercer seus efeitos por influenciar a expressão de genes de resposta precoce, como o fator nuclear (NF)-кB. Nesse contexto, vários estudos mostraram que a deformação tecidual pulmonar ativa o NF-кB, que é transportado para o núcleo, onde se liga a regiões promotoras do DNA, que regulam a produção de IL-6, IL-8, IL1- $\beta$ e TNF- $\alpha{ }^{45}$.

Diferentes estímulos mecânicos também podem alterar a expressão gênica de componentes da matriz extracelular (MEC) ${ }^{46}$. Nesse contexto, Garcia e col. demonstraram que tiras de parênquima pulmonares sadias submetidas a oscilações senoidais com amplitude (estiramento) constante e tensões (estresse) elevadas, aumentam a expressão de RNAm para procolágeno tipo III (PCIII). Adicionalmente, esses autores determinaram a existência de um limiar de estresse acima do qual as células pulmonares expressam RNAm para PCIII ${ }^{46}$.

\section{Translocação de Bactérias e/ou seus Produtos do Pulmão}

A VM lesiva também pode promover a translocação de bactérias e/ou seus produtos do pulmão para o sangue, contribuindo para o desenvolvimento de disfunção de múltiplos órgãos e sistemas (DMOS) ${ }^{47}$. A DMOS também pode ocorrer em função dos seguintes mecanismos: (1) lesão por estresse da barreira alvéolo-capilar (descompartimentalização), (2) alteração na estrutura do citoesqueleto, (3) efeitos nos vasos pulmonares independentemente de ruptura, e, como proposto mais recentemente, (4) o estresse cíclico que impede o reparo da membrana alvéolo-capilar ${ }^{5}$.

A distensão pulmonar total associada com a abertura e colapso cíclico das unidades distais facilitam a translocação de bactérias instiladas intratraquealmente para o sangue ${ }^{47}$, como conseqüência da lesão da barreira alvéolo-capilar, efeito reduzido pela adição de PEEP. Estratégias ventilatórias como $V_{T}$ alto e PEEP igual a zero também podem promover a translocação de endotoxinas do pulmão para a circulação sistêmi$\mathrm{ca}^{48}$. Outro importante fator produzido localmente no pulmão e liberado sistemicamente em função da hiperdistensão alveolar é o Fas ligante. Ele se acumula, na forma solúvel, em vários locais do tecido inflamado, apresentando potencial para desencadear a apoptose de leucócitos, células epiteliais e outras células parenquimatosas ${ }^{49}$.

\section{Apoptose/Necrose}

Quando as células morrem de forma organizada ou semi-organizada pode-se dizer que está ocorrendo apoptose; mas se elas sucumbem com sinais endógenos ou exógenos define-se como necrose ${ }^{5}$. $\mathrm{O}$ balanço entre a apoptose e a necrose pode ser relevante não apenas no reparo da barreira alvéolo-capilar, mas também na reabsorção do exsudato de fibrinas pulmonares, resultando na recuperação da arquitetura e do funcionamento pulmonar normal.

A presença de apoptose foi evidenciada em pulmões com SDRA ventilados com estratégia não lesiva; já a necrose predominou na estratégia ventilatória lesiva sendo acompanhada de apoptose em órgãos distais (rins, intestinos e fígado) ${ }^{50}$.

\section{CONCLUSÃO}

A VM acarreta lesões biofísicas e bioquímicas, podendo causar DMOS e morte. Os mecanismos responsáveis pelo volutrauma, atelectrauma e biotrauma devem ser bem entendidos para que se possa evitar a lesão associada à ventilação mecânica.

\section{REFERÊNCIAS}

01. Tobin MJ - Advances in mechanical ventilation. $\mathrm{N}$ Engl $\mathrm{J}$ Med, 2001;344:1986-1996.

02. Pinhu L, Whitehead T, Evans $\mathrm{T}$ et al - Ventilator-associated lung injury. Lancet, 2003;361:332-340.

03. Han B, Lodyga M, Liu M - Ventilator-induced lung injury: role of protein-protein interaction in mechanosensation. Proc Am Thorac Soc, 2005;2:181-187.

04. The Acute Respiratory Distress Syndrome Network - Ventilation with lower tidal volumes as compared with traditional tidal volumes for acute lung injury and the acute respiratory distress syndrome. N Engl J Med, 2000;342:1301-1308.

05. Dos Santos CC, Slutsky AS - The contribution of biophysical lung injury to the development of biotrauma. Ann Rev Physiol, 2006;68:585-618.

06. Dreyfuss D, Basset $G$, Soler $P$ et al - Intermittent positive-pressure hyperventilation with high inflation pressures produces pulmonary microvascular injury in rats. Am Rev Respir Dis, 1985;132:880-884.

07. Dreyfuss D, Soler P, Basset G et al - High inflation pressure pulmonary edema. Respective effects of high airway pressure, high tidal volume, and positive end-expiratory pressure. Am Rev Respir Dis, 1988;37:1159-1164.

08. Bachofen M, Weibel ER - Structural alterations of lung parenchyma in the adult respiratory distress syndrome. Clin Chest Med, 1982;3:35-56.

09. Garcia CSNB, Rocco PRM, Zin WA - Understanding the Mechanism of Ventilator-Induced Lung Injury, em: Gullo A, Berlot G - Perioperative and Critical Care Medicine. $1^{\text {st }}$ Ed, Milão, Springer, 2005;43-59.

10. Webb HH, Tierney DF - Experimental pulmonary edema due to intermittent positive pressure ventilation with high inflation pressures. Protection by positive end-expiratory pressure. Am Rev Respir Dis, 
1974;110:556-565.

11. John $\mathrm{E}, \mathrm{McDevitt} \mathrm{M}$, Wilborn $\mathrm{W}$ et al - Ultrastructure of the lung after ventilation. $\mathrm{Br} J$ Exp Pathol, 1982;63:401-407.

12. Tsuno K, Prato P, Kolobow $T$ - Acute lung injury from mechanical ventilation at moderately high airway pressures. J Appl Physiol, 1991;69:956-961.

13. Albert RK, Lakshminarayan $\mathrm{S}$, Hildebrandt $\mathrm{J}$ et al - Increased surface tension favors pulmonary edema formation in anesthetized dog's lungs. J Clin Invest, 1979;63:1015-1018.

14. Nolop KB, Maxwell DL, Royston D et al - Effect of raised thoracic pressure and volume on 99mTc-DTPA clearance in humans. J Appl Physiol, 1986;60:1493-1497.

15. Dreyfuss D, Soler P, Saumon G - Mechanical ventilation-induced pulmonary edema. Interaction with previous lung alterations. Am J Respir Crit Care Med, 1995;151:1568-1575.

16. Albert RK, Lakshminarayan S, Kirk W et al - Lung inflation can cause pulmonary edema in zone I of in situ dog lungs. J Appl Physiol, 1980;49:815-819.

17. Pattle RE - Properties, function and origin of the alveolar lining layer. Nature, 1955; $175: 1125-1126$

18. O'Brodovich $\mathrm{H}$, Coates $\mathrm{G}$, Marrin M - Effect of inspiratory resistance and PEEP on 99mTc-DTPA clearance. J Appl Physiol, 1986;60:1461-1465.

19. Kim KJ, Crandall ED - Effects of lung inflation on alveolar epithelial solute and water transport properties. J Appl Physiol, 1982;52:1498-1505.

20. Lecuona $E$, Saldias $F$, Comellas A et al - Ventilator-associated lung injury decreases lung ability to clear edema in rats. Am J Respir Crit Care Med, 1999;159:603-609.

21. Ito Y, Veldhuizen RA, Yao LJ et al - Ventilation strategies affect surfactant aggregate conversion in acute lung injury. Am J Respir Crit Care Med, 1997; 155:493-499.

22. Lewis JF, Veldhuizen R, Possmayer F et al - Altered alveolar surfactant is an early marker of acute lung injury in septic adult sheep. Am J Respir Crit Care Med, 1994;150:123-130.

23. Veldhuizen RA, McCaig LA, Akino T et al - Pulmonary surfactant subfractions in patients with the acute respiratory distress syndrome. Am J Respir Crit Care Med, 1995;152:1867-1871.

24. Malloy JL, Veldhuizen RA, Lewis JF - Effects of ventilation on the surfactant system in sepsis-induced lung injury. J Appl Physiol, 2000;88:401-408.

25. Parker JC, Hernandez LA, Longenecker GL et al - Lung edema caused by high peak inspiratory pressure in dogs. Role of increased microvascular filtration pressure and permeability. Am Rev Respir Dis, 1990;142:321-328.

26. Bouhuys A - Physiology and musical instruments. Nature, 1969;221:1199-1204.

27. Hernandez LA, Peevy KJ, Moise AA et al - Chest wall restriction limits high airway pressure-induced lung injury in young rabbits. J Appl Physiol, 1989;66:2364-2368.

28. Carlton DP, Cummings JJ, Scheerer RG et al - Lung overexpansion increases pulmonary microvascular protein permeability in young lambs. $J$ Appl Physiol, 1990; 69:577-583.

29. Gattinoni L, Caironi P, Pelosi P et al - What has computed tomography taught us about the acute respiratory distress syndrome? Am J Respir Crit Care Med, 2001;164:1701-1711.

30. Tremblay LN, Slutsky AS - Ventilator-induced injury: from barotrauma to biotrauma. Proc Assoc Am Physicians, 1998;110:482-488.

31. Ranieri VM, Suter PM, Tortorella $C$ et al - Effect of mechanical ventilation on inflammatory mediators in patients with acute respiratory distress syndrome: a randomized controlled trial. JAMA, 1999;282:54-61.

32. Tremblay L, Valenza F, Ribeiro SP et al - Injurious ventilatory strategies increase cytokines and c-fos $\mathrm{m}$-RNA expression in an isolated rat lung model. J Clin Invest, 1997;99:944-952.

33. Ricard JD, Dreyfuss D, Saumon G - Production of inflammatory cytokines in ventilator-induced lung injury: a reappraisal. Am J Respir Crit Care Med, 2001;163:1176-1180.

34. Bregeon F, Delpierre S, Chetaille B et al - Mechanical ventilation affects lung function and cytokine production in an experimental model of endotoxemia. Anesthesiology, 2005;102:331-339.

35. Chiumello D, Pristine G, Slutsky AS - Mechanical ventilation affects local and systemic cytokines in an animal model of acute respiratory distress syndrome. Am J Respir Crit Care Med, 1999;160:109-116.

36. Conrad SA, Zhang S, Arnold TC et al - Protective effects of low respiratory frequency in experimental ventilator-associated lung injury. Crit Care Med, 2005;33:835-840.

37. Hotchkiss JR Jr, Blanch L, Murias G et al - Effects of decreased respiratory frequency on ventilator-induced lung injury. Am J Respir Crit Care Med, 2000;161:463-468.

38. Wang SH, Wei TS - The outcome of early pressure-controlled inverse ratio ventilation on patients with severe acute respiratory distress syndrome in surgical intensive care unit. Am J Surg, 2002;183:151-155.

39. Casetti AV, Bartlett RH, Hirschl RB - Increasing inspiratory time exacerbates ventilator-induced lung injury during high-pressure/high-volume mechanical ventilation. Crit Care Med, 2002;30:2295-2299.

40. Garcia CSB, Abreu S, Lassance R et al - Mechanical stress caused by high inspiratory airflow in normal lungs. Proc Am Thorac Soc, 2006; 3 (Suppl1): 378.

41. Peevy KJ, Hernandez LA, Moise AA et al - Barotrauma and microvascular injury in lungs of nonadult rabbits: effect of ventilation pattern. Crit Care Med, 1990;18:634-637.

42. Rich PB, Reickert CA, Sawada S et al - Effect of rate and inspiratory flow on ventilator-induced lung injury. J Trauma, 2000;49:903-911.

43. Gattinoni L, Carlesso E, Cadringher P et al - Physical and biological triggers of ventilator-induced lung injury and its prevention. Eur Respir J, 2003;47:(Suppl);15S-25S.

44. Garcia CS, Prota LF, Morales MM et al - Understanding the mechanisms of lung mechanical stress. Braz J Med Biol Res, 2006;39:697-706.

45. Schwartz MD, Moore EE, Moore FA et al - Nuclear factor-kappa B is activated in alveolar macrophages from patients with acute respiratory distress syndrome. Crit Care Med, 1996;24:1285-1292.

46. Garcia CS, Rocco PR, Facchinetti LD et al - What increases type III procollagen mRNA levels in lung tissue: stress induced by changes in force or amplitude? Respir Physiol Neurobiol, 2004;144:59-70.

47. Verbrugge SJ, Sorm V, van 't Veen A et al - Lung overinflation without positive end-expiratory pressure promotes bacteremia after experimental Klebsiella pneumoniae inoculation. Intensive Care Med, 1998;24:172-177.

48. Murphy DB, Cregg N, Tremblay L et al - Adverse ventilatory strategy causes pulmonary-to-systemic translocation of endotoxin. Am J Respir Crit Care Med, 2000;162:27-33.

49. Plotz FB, Slutsky AS, van Vught AJ et al - Ventilator-induced lung injury and multiple system organ failure: a critical review of facts and hypotheses. Intensive Care Med, 2004;30:1865-1872.

50. Imai $Y$, Parodo J, Kajikawa $O$ et al - Injurious mechanical ventilation and end-organ epithelial cell apoptosis and organ dysfunction in an experimental model of acute respiratory distress syndrome. JAMA, 2003;289:2104-2112. 